\title{
Effect of vertical seismic motion on the dynamic response and instantaneous liquefaction in a two-layer porous seabed
}

\author{
Wei-Yun Chen ${ }^{\mathrm{a}}$, Zhi-Hua Wang ${ }^{\mathrm{a}}$, Guo-Xing Chen ${ }^{\mathrm{a}}$, Dong-Sheng Jeng ${ }^{\mathrm{b}}$, Ming $\mathrm{Wu}^{\mathrm{c}}$, \\ Hong-Yi Zhao d,* \\ a Institute of Geotechnical Engineering, Nanjing Tech University, Nanjing 210009, China \\ ${ }^{\mathrm{b}}$ Griffith School of Engineering, Griffith University Gold Coast Campus, Queensland QLD \\ 4222, Australia \\ ${ }^{\mathrm{c}}$ key laboratory of Western China's Mineral Resources of Geological Engineering, Ministry of \\ Education, Chang'an University, Xi'an 710054, China \\ ${ }^{\mathrm{d}}$ College of Harbor, Coastal and Offshore Engineering, Hohai University, Nanjing 210098, China
}

\section{ABSTRACT}

The evaluation of seismic-induced response in the porous seabed is a fundamental problem in geotechnical and coastal engineering. Although the ground motions generally include both horizontal and vertical components, most previous theoretical investigations assumed vertically propagating shear waves in a horizontally layered soil-rock system and simply ignored the effect of site response to vertical earthquake motion. In this paper, the dynamic response and instantaneous liquefaction of porous seabed, induced by vertical earthquake loading, is studied using an analytical method. The seabed is treated as a two-layer poro-elastic medium and characterized by the fully dynamic formulation of Biot theory. The analytical solutions for the response variables, such as induced displacement, pore pressure and vertical effective stress, are respectively derived and the mechanism of instantaneous liquefaction in the liquefiable sediment is investigated based on the excess pore pressure criterion. A set of parametric analysis is carried out to discuss the effects of seawater, seabed and earthquake parameters on the seismic response and maximum liquefaction depth. It is worth noting that the properties of surface seabed layer have significant influence on the seismic response and consequently the potential stability of the seabed, which is important in the analysis of foundations for offshore structures.

Keywords: Layered seabed, Seismic response, Instantaneous liquefaction, Pore pressure, Vertical earthquake. 


\section{Introduction}

In recent decades, more and more offshore structures, such as submarine tunnels, cross-sea bridges and oil platforms, have been constructed in the continental margins of the worldwide oceans. In the design of coastal structures, the instability of foundation under the environmental loading has long been an important issue. Generally, particular concerns are often addressed on two types of conventional loading in offshore area: ocean waves and earthquake. The effect of ocean-wave loading on the seabed and marine structures has been extensively studied. Yet, attention was rarely paid on the dynamic behavior of seabed under seismic action in existing literatures, which is surprising in view of the failures of seabed and marine structure, as reported in the many earthquakes [1-4]. Such accidents and their destructive consequences show that it is essential to consider seismic-induced response in the seabed when designing the foundations of marine structures in active seismic regions.

In the marine environment, liquefaction plays an important role around or beneath offshore structures, as it often occurs in saturated or nearly saturated granular materials, like cohesionless seabed soils. Ocean-waves induced response and instability of seabed have already attracted great attention in geotechnical engineering and coastal engineering in the last few decades [5-8]. It is generally accepted that the liquefaction of the seabed is closely associated with pore pressure change and the resultant degradation of the soil's macroscopic properties. Two mechanisms for wave-induced liquefaction have been reported in previous laboratory and theoretical studies, depending on how the excess pore pressure is generated $[9,10]$. One mechanism is wave-induced (residual) liquefaction, which results from the pore pressure accumulation due to the volumetric compaction under cyclic wave loading [11]. Another so-called transient liquefaction will occur when the instantaneous value of the (oscillatory) pore pressure directly exceeds a certain mean level and the vertical effective stress vanishes.

In addition to ocean-waves, seismic loading is another external factor leading to liquefaction in the seabed. Actually, the full elastic seismic wavefield that propagates through an isotropic earth consists of shear and compressional waves, depending on the vibrating direction of the substrate bedrock. In addition, the dynamic response of marine waters under the incidence of seismic events is significant [12] and could also be viewed as another important underlying cause of liquefaction 
1 in the seabed. So far, considerable interest has been paid to study the liquefaction triggered by

2 horizontal motion associated with the propagation of shear waves $[13,14]$. These studies

3 concluded that the liquefaction was associated with substantial soil non-linearity and build-up of

4 pore pressure. On the other hand, the analysis on vertical motion directly related with

5 compressional wave is quite limited, especially for the seabed. Based on the observation in in-situ

6 field and analytical solutions for various response, Yang [15] revealed that the effect of vertical

7 motion on liquefaction was not negligible and the effect was dependent on the saturation condition.

8 Less nonlinearity of soil was found to affect the vertical motion involving mainly the propagation

9 of compressional wave [16]. Based on dynamic Biot's equations for the porous seabed, Ye [17]

10 and Ye and Wang [18] adopted the FEM numerical model to investigate both the vertical and

11 horizontal seismic response of a composite breakwater and its seabed under the pacific coast of

12 Tohoku earthquake.

13 In addition, most of the previous investigations modeled seabed as uniform single layer and of either finite or infinite thickness [10,19]. In the real ocean environment, the seabed may consist of two or more layers with different soils and only a few dynamic researches attempted to treat the porous seabed as non-homogenous layered medium [20-23]. As summarized above, although the dynamic response of porous seabed has caught extensive concern, to our knowledge, the corresponding literature for the seismic response of a layered seabed has not been published to date.

Aimed at this goal, an analytical study is conducted to determine the response of layered seabed induced by vertical seismic loading and how it differs from that of the conventional single-layer approach. The analytical solutions for the response variables, such as induced displacement, pore pressure and vertical effective stress, are derived based on the fully dynamic formulation of governing equations. Then the mechanism of potentially instantaneous liquefaction in the liquefiable sediment is investigated. A set of parametric analysis is carried out to discuss the effect of related parameters on the maximum liquefaction depth.

\section{Dynamic response of seabed: governing equations}

In this paper, the marine sediment is considered as a mixture consisting of three phases: a solid 
1 phase that forms skeletal frame, a liquid phase that occupies a major portion of pore space, and a

2 gas phase that occupies a small portion of pore space. The air phase is assumed to be fully

3 dissolved in the fluid phase, constituting a single compressible fluid. To develop the governing

4 equations for the dynamic response of the porous seabed, three relationships should be included:

5 stress-strain constitutive relation, momentum balance equations and mass balance equation. In most previous studies $[10,17,18]$, the so-called " $u-p$ " approximate formulation, which ignores the

7 displacement of pore fluid relative to soil particles, was used in the dynamic analysis. For the

8 seismic issue in relatively high frequency range, however, all the inertial terms should be considered to obtain more accurate solutions. This formulation is called fully dynamic formulation

10 [19]. The dynamic Biot's equations [24] developed by Zienkiewicz et al. [25] are briefly summarized to describe the dynamic response of the porous seabed.

The effective stress controlling the deformation of porous medium is written as

$$
\sigma_{i j}=\sigma_{i j}^{\prime}+\delta_{i j} p \quad(i, j=x, z)
$$

where $\sigma_{i j}$ is total stress; $\sigma_{i j}^{\prime}$ is effective stress; $\delta_{i j}$ is Kronecker delta; $p$ is pore pressure. Compression is taken as positive for the pore pressure.

The strain $\varepsilon_{i j}$ is defined as:

$$
\varepsilon_{i j}=\frac{1}{2}\left(u_{i, j}+u_{j, i}\right)
$$

where $u_{i, j}$ and $u_{j, i}$ are the derivatives of the soil skeleton displacement with respect to spatial coordinates.

Using the Lame's parameters, $\lambda$ and $\mu$, the effective stresses is written as

$$
\sigma_{i j}^{\prime}=\lambda \varepsilon_{k k} \delta_{i j}+2 \mu \varepsilon_{i j}
$$

where $\mu$ is also called shear modulus of soil and $\lambda=2 \mu v /(1-2 v)$ with the Poisson's ratio $v$.

The overall equilibrium of the system and the equilibrium of the pore fluid are respectively written as

$$
\sigma_{i j, j}+\rho g_{i}-\rho \ddot{u}_{i}-\rho_{f} \ddot{\bar{w}}_{i}=0
$$


where $g_{i}$ is the body force acceleration, $\rho$ is total density of the porous medium, $\rho=(1-n) \rho_{s}+n \rho_{f}, \quad \rho_{s}$ is the density of the solid skeleton, $n$ is the soil porosity; $\rho_{f}$ is density for the liquid, $k_{f}$ denotes the coefficient of hydraulic conductivity. $\ddot{u}_{i}$ denotes the acceleration of solid skeleton, $\ddot{\bar{w}}_{i}$ is the average pore fluid acceleration relative to solid frame. Noting that the average pore fluid relative displacement is denoted as [26]

$$
w_{i}=n\left(W_{i}-u_{i}\right)
$$

where $u_{i}$ is the displacement of the solid skeleton, $W_{i}$ is the total fluid displacement.

The law of conservation of mass yields

$$
\dot{\varepsilon}_{k k}+\dot{\bar{w}}_{i, i}-n \beta \dot{p}=0
$$

where $\beta$ is the compressibility of the pore fluid account for slight unsaturation through the degree of saturation $S_{r}$ as [27]

$$
\beta=\frac{1}{K_{w}}+\frac{1-S_{r}}{p_{w 0}}
$$

where $K_{w}$ is the true bulk modulus of water and $p_{w 0}$ is the pore water pressure, i.e., $p_{w o}=\rho_{f} g d, d$ is the depth of seawater.

\section{Analytical solutions for the seismic response variables}

In this study, we consider a two-layered porous seafloor with a finite thickness $L$ above a half-space (i.e., bedrock), as shown in Fig. 1. The flexibility of an underlying half-space could be influential to earthquake behaviors of the porous seabed $[28,29]$. For the seismic problem under consideration, the underlying half-space is assumed to be impermeable and rigid bedrock, the ground motion of which is counted as seismic input [30]. This assumption is widely accepted in seismic analysis of geotechnical earthquake engineering. The relative ratio of lower layer thickness, $m$, is adopted to define various layer thicknesses. The depth of the overlying seawater is assumed to be $d$. The seabed material is treated as an elastic porous media with linear elasticity. The positive direction of $z$ axis points vertically upwards and the $x$ axis is assumed along 
1 horizontal direction. As an acceptable assumption in geotechnical earthquake engineering, site

2 response to earthquakes is regarded as the consequence of vertical propagation of shear and compressional waves induced by multidirectional earthquake motions originating primarily from the underlying bedrock [15]. To simulate the seismic ground motion, the steady-state displacement excitation is specified vertically at the base of the soil deposit.

In this case, the compressional waves are generated vertically in the seabed and the vibration direction of the particles is consistent with the direction of wave propagation. Accordingly, the variables $u$ and $W$ represent the solid skeleton displacement and total fluid displacement in the compression direction (i.e., $z$ axis direction). Neglecting the body force terms and combining equations (1) (3), (6) and (7), the governing equations (4) and (5) can be rewritten as

$$
\begin{gathered}
\left(\lambda+2 \mu+\frac{1-n}{n \beta}\right) \frac{\partial^{2} u}{\partial z^{2}}+\frac{1}{\beta} \frac{\partial^{2} W}{\partial z^{2}}+\left(\rho_{f} n-\rho\right) \frac{\partial^{2} u}{\partial t^{2}}-\rho_{f} n \frac{\partial^{2} W}{\partial t^{2}}=0 \\
\frac{1-n}{n \beta} \frac{\partial^{2} u}{\partial z^{2}}+\frac{1}{\beta} \frac{\partial^{2} W}{\partial z^{2}}-\frac{n \rho_{f} g}{k_{f}}\left(\frac{\partial W}{\partial t}-\frac{\partial u}{\partial t}\right)-\rho_{f} \frac{\partial^{2} W}{\partial t^{2}}=0
\end{gathered}
$$

Based on equations (9) and (10), a four-order governing equation for the soil skeleton displacement can be deduced as

$$
\frac{\partial^{4} u}{\partial t^{4}}+a_{1} \frac{\partial^{3} u}{\partial t^{3}}+a_{2} \frac{\partial^{4} u}{\partial t^{2} \partial z^{2}}+a_{3} \frac{\partial^{3} u}{\partial t \partial z^{2}}+a_{4} \frac{\partial^{4} u}{\partial z^{4}}=0
$$

in which

$$
\begin{array}{ll}
a_{1}=-\frac{n \rho g}{k_{f}\left(n \rho_{f}-\rho\right)} & a_{2}=\frac{n \rho+\rho_{f}[(\lambda+2 \mu) n \beta+1-2 n]}{\rho_{f} n \beta\left(n \rho_{f}-\rho\right)} \\
a_{3}=\frac{g[(\lambda+2 \mu) n \beta+1]}{\beta k_{f}\left(n \rho_{f}-\rho\right)} & a_{4}=-\frac{\lambda+2 \mu}{\beta \rho_{f}\left(n \rho_{f}-\rho\right)}
\end{array}
$$

Considering all the response variables to be of the form, $F=F_{0}(z) e^{\mathrm{i}(\omega t-z k)}$, where $F_{0}$ represents the amplitude of the corresponding response variable, such as the solid and fluid displacements, pore pressure and stress in the soils; $i$ is the root of $-1, t$ is time, $\omega$ is the angular frequency, $k$ is a complex number. Then equation (11) is rewritten as

$$
a_{4} k^{4}+\left(a_{2} \omega^{2}-\mathrm{i} a_{3} \omega\right) k^{2}+\omega^{4}-\mathrm{i} a_{1} \omega^{3}=0
$$

The roots of equation (12) are assumed to be $\pm k_{p 1}$ and $\pm k_{p 2}$ and $\left|\operatorname{Re}\left(k_{p 1}\right)\right|<\left|\operatorname{Re}\left(k_{p 2}\right)\right|$, where $\operatorname{Re}()$ 
represents the real part of the corresponding complex root. In soil dynamics, $k_{p 1}$ and $k_{p 2}$ denote the complex wave numbers for two types of compressional waves, namely P1 wave and P2 wave in the saturated soils [24]. The real and imaginary part of complex wave numbers can also be used to calculate the wave speed and attenuation coefficient of the waves propagating in the seabed, respectively.

The general solution for earthquake-induced displacement in equation (11) is assumed to take the form as

$$
\text { the form as }
$$

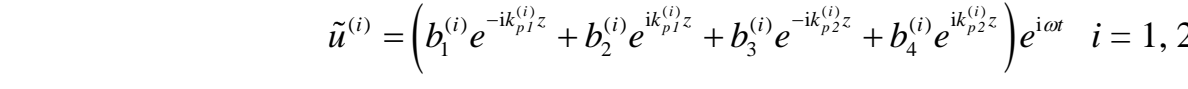

Here superscript indices 1 and 2 refer to the first and second layers, respectively. Equation (13) indicates that the induced displacements in the soils are associated with the propagation characteristics of the waves in different seabed layers. Using the same operations and with the aid of equations (6), the induced relative fluid displacement in the seabed takes the similar form as

$$
\tilde{w}^{(i)}=\left(\eta_{1}^{(i)} b_{1}^{(i)} e^{-\mathrm{i} k_{p 1}^{(i)} z}+\eta_{2}^{(i)} b_{2}^{(i)} e^{i k_{p 1}^{(i)} z}+\eta_{3}^{(i)} b_{3}^{(i)} e^{-\mathrm{ik} p_{2}^{(i)} z}+\eta_{4}^{(i)} b_{4}^{(i)} e^{\mathrm{i} k_{p 2}^{(i)} z}\right) e^{\mathrm{i} \omega t} \quad i=1,2
$$

with

$$
\begin{aligned}
& \eta_{1}^{(i)}=\eta_{2}^{(i)}=\frac{\left(\lambda^{(i)}+2 \mu^{(i)}+\frac{1}{n^{(i)} \beta^{(i)}}\right) k_{p 1}^{(i) 2}-\omega^{2} \rho^{(i)}}{\omega^{2} \rho_{f}^{(i)}-\frac{1}{n^{(i)} \beta^{(i)}} k_{p 1}^{(i) 2}} \\
& \eta_{3}^{(i)}=\eta_{4}^{(i)}=\frac{\left(\lambda^{(i)}+2 \mu^{(i)}+\frac{1}{n^{(i)} \beta^{(i)}}\right) k_{p 2}^{(i) 2}-\omega^{2} \rho^{(i)}}{\omega^{2} \rho_{f}^{(i)}-\frac{1}{n^{(i)} \beta^{(i)}} k_{p 2}^{(i) 2}}
\end{aligned}
$$
are calculated using the volumetric strains as

$$
\begin{gathered}
\tilde{p}^{(i)}=\frac{\mathrm{i}}{n^{(i)} \beta^{(i)}}\left(\begin{array}{l}
b_{1}^{(i)}\left(1+\eta_{1}^{(i)}\right) k_{p 1}^{(i)} e^{-\mathrm{i} k_{p 1}^{(i)} z}-b_{2}^{(i)}\left(1+\eta_{2}^{(i)}\right) k_{p 1}^{(i)} e^{\mathrm{i} k_{p 1}^{(i)} z} \\
+b_{3}^{(i)}\left(1+\eta_{3}^{(i)}\right) k_{p 2}^{(i)} e^{-\mathrm{i} k_{p 2}^{(i)} z}-b_{4}^{(i)}\left(1+\eta_{4}^{(i)}\right) k_{p 2}^{(i)} e^{\mathrm{i} k_{p 2}^{(i) z}}
\end{array}\right) e^{\mathrm{i} \omega t} \\
\tilde{\sigma}_{z z}^{\prime(i)}=\left(\lambda^{(i)}+2 \mu^{(i)}\right) \mathrm{i}\left(-k_{p 1}^{(i)} b_{1}^{(i)} e^{-\mathrm{i} k_{p 1}^{(i)} z}+k_{p 1}^{(i)} b_{2}^{(i)} e^{\mathrm{i} k_{p 1}^{(i)} z}-k_{p 2}^{(i)} b_{3}^{(i)} e^{-\mathrm{i} k_{p 2}^{(i) z}}+k_{p 2}^{(i)} b_{4}^{(i)} e^{\mathrm{i} k_{p 2}^{(i)} z}\right) e^{\mathrm{i} \omega t}
\end{gathered}
$$

As demonstrated in equations (13) (16), the earthquake-induced displacements as well as pore pressure and effective stress in the seabed are associated with the independent contributions from 


\section{Boundary conditions}

A set of boundary conditions for the problem of seismic response in two-layer seabed are introduced to solve the coefficients, $b_{j}^{(1)}$ and $b_{j}^{(2)}(j=1-4)$ in equations (13) and (14). Firstly, at seabed surface $(z=L)$, i.e., the interface between seawater and seabed, the shear stress is assumed to be negligible for the ideal inviscid seawater. In addition, the vertical effective stress vanishes and the pore pressure in the soil, $\tilde{p}^{(1)}$, equals to the seismically induced seawater pressure above the surface, $p_{w}$, which is given later in Eq. (19). Considering that the pore pressure is taken as positive for tensile stress, as denoted in equation (1), these boundary conditions can be written as

$$
\tilde{p}^{(1)}=p_{w}, \quad \tilde{\sigma}_{z z}^{\prime(1)}=0
$$

The compatibility between the normal displacement of upper seawater $w_{u}$ and the normal pore fluid movement in and out of the skeletal frame is given by

$$
w_{u}=(1-n) \tilde{u}^{(1)}+n \tilde{W}^{(1)}
$$

where $\tilde{W}^{(1)}$ is the earthquake-induced total fluid displacement in the upper soil layer.

It should be noted that the compressibility of overlying seawater could be influential to the dynamic responses of seabed in some cases [29]. In fact, the different manifestations of compressibility of the seawater, such as the generation of hydroacoustic wave and forced oscillation, are shown to correspond to the cases with different frequency ranges, based on the offshore observatory data [31]. The low frequency phenomena can be regarded approximately as the incompressible water, whereas high frequency phenomena are attributed to the water compressibility. It is revealed that bottom dynamic pressure induced by the vibration of seabed, i.e., $p_{w}$, follows the bottom acceleration within the frequency band of 'forced oscillation' [31-33] as

$$
p_{w}=\rho_{f} d \ddot{w}_{u} \text { for } f \leq f_{\mathrm{c}}
$$

where $f$ is the frequency and $f=\omega / 2 \pi, f_{\mathrm{c}}$ is the characteristic frequency of the fundamental acoustic resonance of seawater assuming the rigid ocean-bottom $\left(f_{c}=c_{w} / 4 d\right), c_{w}$ is the speed of the compression wave in fluid. Equation (19) is associated with the case of rigid ocean-bottom, so the value of $f_{c}$ will be larger for the nonrigid ocean-bottom in this paper. In addition, for most of the 
1 offshore structures and installations this paper focuses on, the seawater is not very deep. Thus

2 most of the dominant frequency range of seaquake loads is lower than the value of $f_{\mathrm{c}}$ and the 3 pressure response calculated using equation (19) is valid.

4 In addition, the pore pressure, soil displacements, and effective stresses are assumed to be 5 continuous at the interface between the upper and lower layers, i.e., $z=m L$.

$$
\tilde{p}^{(1)}=\tilde{p}^{(2)}, \quad \tilde{\sigma}_{z z}^{(1)}=\tilde{\sigma}_{z z}^{\prime(2)}, \quad \tilde{u}^{(1)}=\tilde{u}^{(2)}, \quad \tilde{w}^{(1)}=\tilde{w}^{(2)}
$$

Finally, the earthquake loading is usually irregular and stochastic in practical seismic record. For the sake of simplification in this analytical study, the vertical excitation with frequency $\omega$ and displacement amplitude $u_{0}$ is assumed to act on the impermeable rigid bottom, i.e., $z=0$, they are

$$
\tilde{u}^{(2)}=u_{0} \mathrm{e}^{\mathrm{i} \omega t}, \quad \tilde{w}^{(2)}=0
$$

By combining the boundary conditions (17), (20) and (21), the coefficients $b_{j}^{(1)}$ and $b_{j}^{(2)}(j=$ 1-4) in equations (13) and (14) can be solved (see Appendix).

\section{Seismic-induced response of porous seabed}

In general, the seismic-induced response, such as displacements, pore pressure and effective stress in seabed, are important to the deformation and stability of seabed near the offshore structures. Hence the responses of nearly saturated two-layer porous seabed are presented and discussed in this section. The effect of the surface layer (i.e., layer 1 in Fig. 1) with distinctive material properties on the response of the whole two-layer seabed is investigated. Special attention is given to the effect of the relative thickness, relative soil permeability and relative shear modulus of the upper cover layer on the dynamic response of the two-layer seabed. The vertical distributions of maximum amplitudes of earthquake-induced soil displacement, relative fluid displacement, pore pressure and vertical effective stress versus relative depth are respectively depicted in Figs 3-6. The induced soil displacement $|\tilde{u}|$ and relative fluid displacement $|\tilde{w}|$ are non-dimensionalized with respect to the displacement amplitude at the seabed bottom $u_{0}$. The induced pore pressure $|\tilde{p}|$ and vertical effective stress $\left|\tilde{\sigma}_{z z}^{\prime}\right|$ are normalized by the absolute hydrostatic water pressure at the seabed surface $p_{w 0}$. The density of soil grain $\rho_{s}=2650 \mathrm{~kg} / \mathrm{m}^{3}$, the density of water $\rho_{f}=1000 \mathrm{~kg} / \mathrm{m}^{3}$ and the bulk modulus of water $K_{w}=2.0 \mathrm{GPa}$. Unless otherwise 
specified, the upper layer is assumed to be coarse sand $\left(k_{f}^{(1)}=5 \times 10^{-2} \mathrm{~m} / \mathrm{s}\right)$ while the lower layer is set as fine sand $\left(k_{f}^{(2)}=10^{-3} \mathrm{~m} / \mathrm{s}\right)$

\subsection{Verification of the present model}

The available literature for the seismic response of seabed is quite limited to date. Yang and Sato [30] studied the seismic vertical amplification on land, without considering the overlying water. Although this analysis is not for the marine environment, it can be used to verify the proposed model in the case that the overlying seawater of non-layered seabed is removed. As shown in Fig. 2, the motion amplification factor at the seabed surface in this limiting case (i.e., $d=$ $0 \mathrm{~m}$ ) is compared with the computation results of Yang and Sato [30]. Here the amplification factor is defined as the ratio of the amplitude of solid displacement at any depth to that at the bottom of seabed. The main parameters for comparison are selected from Yang and Sato [30] as: porosity $n=0.37$; seabed permeability $k_{f}=0.001 \mathrm{~m} / \mathrm{s}$; Poisson's ratio $v=0.3$; shear modulus $\mu=$ $65 \mathrm{MPa}$; seabed thickness $L=15 \mathrm{~m}$ and water depth $d=0 \mathrm{~m}$. The influence of frequency on the amplification factor in both the partly saturated case $\left(S_{r}=98 \%\right)$ and fully saturated case $\left(S_{r}=\right.$ 100\%) are considered. Fig. 2 indicates that the present results agree overall with Yang and Sato [30], especially for the fully saturated seabed $\left(S_{r}=100 \%\right)$ within the common frequency range of undersea earthquake. It should be noted that, in the governing equations in Yang and Sato [30], the parameters accounting for the compressibility of grains are $\alpha$ and $M$; and $\alpha=1-K_{b} / K_{s}$, $M=K_{s}^{2} /\left(K_{d}-K_{b}\right), \quad K_{d}=K_{s}\left[1+n\left(K_{s} / K_{f}-1\right)\right]$, in which $K_{s}, K_{b}$ and $K_{f}$ are the bulk moduli of solid grains, solid skeleton and pore fluid, respectively. When the compressibility of solid grains is neglected, i.e., $K_{s} \approx \infty$, the governing equations in Yang and Sato [30] are identical with equations (9) and (10). As indicated in Zienkiewicz and Shiomi [26], for most soil-like materials, $\alpha$ is indeed very close to unity but may be as low as 0.5 for rock-like materials with a small porosity. Therefore, for the liquefiable soils in the porous seabed in this paper, the compressibility of solid grains is also ignored, which is a widely accepted assumption in soil mechanics. The discrepancy between the present study and Yang and Sato [30] may come from this slightly difference in the formulation for the porous media, i.e., the difference of values of 
2 5.2. Effect of relative thickness ratio

3 Fig. 3 illustrates the influence of relative thickness ratio on the four response variables. The 4 relative ratio of lower layer thickness, $m$, is taken to from 0.5 to 1.0 . In the figures, $m=1$ is the 5 case with no upper layer. In Fig. 3(a), the induced soil displacement is shown to increase with the 6 increase of the thickness of surface layer. It is widely accepted that the movement of pore fluid flowing in porous seabed is directly related to the soil permeability. Since the higher the

8 permeability is, the resistance between the solid skeleton and pore fluid is weaker and the relative 9 fluid displacement becomes greater. The permeability of the surface layer is larger than the lower and hence the induced relative fluid displacement also increase with the thickness of surface layer when $m \geq 0.7$, as shown in Fig. 3(b). It is also noted that the relative fluid displacement decreases to zero quickly in the lower layer. In Fig. 3(c), the presence of the surface layer causes the pore pressure to dissipate slower in the seabed. As depicted in Fig. 3(d), the variation of vertical effective stress with depth in the surface layer tends to less drastic when the surface layer gets thicker.

\subsection{Effect of relative soil permeability}

Fig. 4 presents the influence of relative soil permeability on the seismic-response of the two-layer seabed. The relative ratio of lower layer thickness, $m$, is taken to be 0.8 . The permeability of the surface layer $k_{f}^{(1)}$ is assumed to range from $10^{-5} \mathrm{~m} / \mathrm{s}$ to $10^{-1} \mathrm{~m} / \mathrm{s}$, which covers the common cohesionless marine soils such as sandy gravel, coarse sand and fine sand. The induced soil displacement in Fig. 4(a) and relative fluid displacement in Fig. 4(b) are shown to increase with the permeability of surface layer. The induced pore pressure dissipates more slowly in the seabed with higher permeability. The influence of relative permeability on vertical effective stress is shown to be significant when $k_{f}^{(1)} \geq 1 \times 10^{-4} \mathrm{~m} / \mathrm{s}$.

\subsection{Effect of relative shear modulus}

In Fig. 5, the influence of relative shear modulus on the seismic-response is illustrated. The shear modulus of the surface layer $\mu^{(1)}$ is taken to from 2.5 MPa to $100 \mathrm{MPa}$. When the shear modulus of layer 2 is greater than layer 1, the induced soil displacement increases with the shear 
modulus of surface layer. However, this influence becomes not notable, otherwise. In Fig. 5(b), the induced relative fluid displacement in most depth of the surface layer increases with the shear modulus of surface layer. Both the induced pore pressure in Fig. 5(c) and vertical effective stress in Fig. 5(d) increase with the shear modulus of surface layer. The influence of relative shear modulus on the response becomes less significant when $\mu^{(1)} / \mu^{(2)} \geq 5$.

\subsection{Effect of earthquake frequency}

Fig. 6 shows the vertical distribution of soil displacement, relative fluid displacement, pore pressure and vertical effective stress under the conditions of different earthquake frequencies. The frequency is taken to be $0.5 \mathrm{~Hz}, 1 \mathrm{~Hz}, 2 \mathrm{~Hz}$ and $5 \mathrm{~Hz}$, respectively. In Figs. 6(a) and 6(b), the displacements change more significant from the seabed bottom to its upper surface with the increasing of frequency. This finding is certainly reasonable from an intuitive point of view, since the case of sufficiently low frequency will approach to the quasi-static state and the whole seabed will have the same displacement. In most depths of seabed, greater pore pressure and vertical effective stress could be induced under the earthquake of higher frequency, as shown in Figs. 6(c) and $6(\mathrm{~d})$.

To have a better understanding of the influence of frequency, the transfer functions of various responses in different relative depths of seabed are also plotted in Fig. 7. The depths are taken to be $z=L$ (at seabed surface), $z=0.8 L$ (at interface between upper and lower layers), $z=0.6 L$ (in lower layer), respectively. In this case, the speed of P1 wave in the seabed is calculated to be $c_{p l}=$ $333 \mathrm{~m} / \mathrm{s}$. Then the fundamental resonance frequencies of $\mathrm{P} 1$ wave in the seabed layer corresponding to the free (or open) and rigid (or closed) boundaries are: $f_{l}=c_{p l} / 2 d=5.55 \mathrm{~Hz}$ and $f_{2}=c_{p l} / 4 d=2.775 \mathrm{~Hz}$, respectively. As shown in Fig. 7, the fundamental peak frequency is observed as $f_{p l}=3.30 \mathrm{~Hz}$, which is closer to fundamental resonance frequency corresponding to rigid (or closed) boundary (i.e., $f_{2}$ ). This is due to the approximation that the seawater on the seabed layer is assumed to be incompressible and the seawater-seabed boundary is to some degree more similar to the rigid (or closed) boundary.

\section{Instantaneous liquefaction of the seabed}

In the case of nearly saturated seabed with small amount of gas in the pore spaces, the increase 
1 of earthquake-induced excess pore pressure may lead to the decrease of effective stress. When the

2 vertical effective stress becomes zero, instantaneous liquefaction will happen. The phenomenon of

3 instantaneous liquefaction in the porous seabed under ocean waves has been widely discussed [21,

$422,34]$. Jeng [35] reviewed and compared three existing criteria of liquefaction, indicating that the

5 two criteria based on the concept of effective normal stresses are not adequate in the examined cases. Thus the criterion of liquefaction Zen and Yamazaki [9] proposed the based on the excess

7 pore pressure is adopted here as:

8

$$
\left(\gamma_{s}-\gamma_{w}\right)(L-z)-\left[\tilde{p}(t)-\tilde{p}_{b}(t)\right] \leq 0
$$

where $\gamma_{s}$ is the unit weight of soil; $\gamma_{w}$ is the unit weight of water; $\tilde{p}(t)$ is the seismic-induced pore pressure in the seabed; $\tilde{p}_{b}(t)$ is the induced dynamic pressure of seawater at the seabed surface $(\mathrm{z}=L)$; The first term is the in-situ effective stress and the second term denotes the excess pore pressure generated in the seabed. This criterion of liquefaction is introduced here to estimate the liquefied state in a porous seabed induced by vertical earthquake loading. The distribution of liquefied soils is related to the relative magnitudes of excess pore pressure and initial effective weight of overlying soils.

Fig. 8 depicts the influence of relative shear modulus on the variation of maximum liquefaction depth with relative thickness ratio. It is shown that the deeper liquefaction will occur in the case of greater shear modulus of the surface layer. Moreover, as the value of $m$ approaches 1.0, the effect of relative shear modulus becomes more insignificant. The influence of seabed thickness on liquefaction depth is demonstrated in Fig. 9. The depth of seawater is assumed to be $12 \mathrm{~m}$. When the two-layer seabed is relatively thin (such as $L=10 \mathrm{~m}$ ), the whole seabed will be liquefied. The depth of liquefaction is shown to increase with the increase of the thickness of seabed. Also, the influence of seawater depth is shown in Fig. 10. The thickness of the seabed is taken to be $30 \mathrm{~m}$. The seabed is more apt to be liquefied under the deeper seawater, just as expected.

The influence of seismic frequency on the maximum liquefaction depth is significant, as depicted in Fig. 11. The liquefaction depth is demonstrated to increase with frequency until the whole seabed is liquefied. As shown in the criterion of liquefaction based on the excess pore pressure, i.e., equation (22), when the excess pore pressure generated in the seabed is greater than 
1 in-situ effective stress, the soils will be liquefied. To have a better insight into the influence of

2 frequency, the variation of $\left[\tilde{p}(t)-\tilde{p}_{b}(t)\right]-\left(\gamma_{s}-\gamma_{w}\right)(L-z)$ with frequency $f$ at different depths of the

3 seabed is illustrated in Fig. 12. The instantaneous liquefaction is predicted to occur when the value

4 exceeds zero. Fig. 12 shows that shallow seabed is more likely to be liquefied at the same 5 frequency.

6 Fig. 13 illustrates the variation of maximum liquefaction depth $d_{l}$ with relative thickness ratio $m$

7 for different relative permeabilities. Distinct values of permeability could represent various types

8 of marine soils. Here the lower layer is set as sand seabed with $k_{f}^{(2)}=10^{-3} \mathrm{~m} / \mathrm{s}$ while the

9 permeability of surface layer $k_{f}^{(1)}$ is taken to be from $10^{-4} \mathrm{~m} / \mathrm{s}$ to $5 \times 10^{-2} \mathrm{~m} / \mathrm{s}$, covering the

10 common cohesionless marine soils. As shown in Fig. 13, increasing the permeability of surface

11 layer is an effective way to reduce the liquefaction depth. Although the presence of the

12 cohesiveless surface layer seems to reduce the liquefaction, the depth of liquefaction does not 13 always decrease with the increase of relative thickness of surface layer, especially when the 14 relative thickness ratio $m$ is less than a threshold value. In this case, the threshold value of $m$ is 15 shown to be about 0.85 for $k_{f}^{(1)}=5 \times 10^{-2} \mathrm{~m} / \mathrm{s}$ and 0.92 for $k_{f}^{(1)}=1 \times 10^{-2} \mathrm{~m} / \mathrm{s}$. On the other hand, when the permeability of surface layer is smaller, the liquefaction depth only increases slightly.

From an engineering viewpoint, replacing some of the original seabed or adding a new layer with different soils is a traditional approach to prevent the seabed. Fig. 14 shows the relationship between liquefaction depth and layer thickness using different treatments: adding a new layer upon the monolayer seabed and replacing (surface) part of the original seabed with different soils. The saturation degree of the seabed is taken to be $99 \%$ and $100 \%$, respectively. It is demonstrated that the partial saturation could increase the risk of liquefaction. By comparison, placing a newly more-permeable surface layer is a more effective way in potentially preventing liquefaction.

\section{Conclusion}

The influence of vertical earthquake loading on the response in porous seabed, which has traditionally been ignored in geotechnical earthquake engineering, is brought into focus in this study. The effects of thickness ratio, soil permeability, shear modulus and earthquake frequency on 
1 the induced response variables (such as soil displacement, relative fluid displacement, pore

2 pressure and vertical effective stress) are respectively presented. Subsequently, a parametric study

3 is carried out on the potentially instantaneous liquefaction of the two-layer seabed. The following

4 conclusions of practical significance emerge from our study:

5 (1) The layered structure of seabed has significant effect on the seismic response and instantaneous liquefaction of seabed. Moreover, the relative thickness ratio has evident effect on the response in the upper layer.

8 (2) The increase of relative soil permeability in the upper layer could increase of both soil displacement and relative fluid displacement and also decrease the maximum liquefaction depth in the seabed.

(3) When the shear modulus of surface layer is smaller than the lower layer, the induced soil displacement, pore pressure and vertical effective stress will increase with the shear modulus of surface layer. Greater relative shear modulus always decreases the liquefaction depth.

(4) The fluctuation of seismic response with depth becomes more marked as the earthquake frequency increases. As expected, the instantaneous liquefaction of seabed will occur when a certain frequency is reached and the depth of liquefaction will increase with the frequency until the whole seabed is liquefied.

(5) Even if the degree of saturation is only slightly below full saturation, the depth of instantaneous liquefaction will increase significantly. Thus it should be noted that a saturated model will always underestimate the potential earthquake-induced damage in the offshore engineering.

(6) From an engineering viewpoint, compared to replacing surface soils of the original seabed, adding a new layer of soil with higher permeability upon the original seabed seems to be a more effective method to protect the seabed from being liquefied.

\section{ACKNOWLEDGEMENT}

The authors would like to acknowledge the financial support provided by the National Natural Science Foundation of China (No. 41502285, 51678299, 41402245), Natural Science Foundation of Jiangsu Province (No. BK20150952). 


\section{References}

[1] Ávila-Carrera R, Flores-Guzmán N, Olivera-Villaseñor E, Rodríguez-Castellanos A, Rodríguez-Sánchez JE. Seismic amplifications from offshore to shore. Appl Ocean Res. 2015; 53: 200-207.

[2] Papatheodorou G, Ferentinos G. Submarine and coastal sediment failure triggered by the 1995, Ms $=6.1 \mathrm{R}$ Aegion earthquake, Gulf of Corinth, Greece. Mar Geol. 1997; 137(3): 287-304.

[3] Sumer BM, Kaya A, Hansen NEO. Impact of liquefaction on coastal structures in the 1999 Kocaeli, Turkey earthquake. Proceedings, The $20^{\text {th }}$ International offshore and polar engineering conference. Japan. 2002; 12: 504-511.

[4] Noda A, Katayama H, Sagayama T, Suga K, Uchida Y, Satake K, Abe K, Okamura Y. Evaluation of tsunami impacts on shallow marine sediments: An example from the tsunami caused by the 2003 Tokachi-oki earthquake, northern Japan. Sediment Geol. 2007; 200(3): 314-327.

[5] Yamamoto T, Koning HL, Sellmeijer H, Hijum EV. On the response of a poro-elastic bed to water waves. J Fluid Mech. 1978; 87(1): 193-206.

[6] Mei CC, Foda MA. Wave-induced responses in a fluid-filled poro-elastic solid with a free surface-a boundary layer theory. Geophys. J. Int. 1981; 66(3): 597-631.

[7] Jeng DS, Lin YS. Poroelastic analysis of the wave-seabed interaction problem. Comput. Geotech. 2000; 26(1): 43-64.

[8] Gao FP, Jeng DS, Sekiguchi H. Numerical study on the interaction between non-linear wave, buried pipeline and non-homogenous porous seabed. Comput. Geotech. 2003; 30(6): 535-547.

[9] Zen K, Yamazaki H. Oscillatory pore pressure and liquefaction in seabed induced by ocean waves. Soil Found. 1990; 30(4): 161-179.

[10] Zhao HY, Jeng DS, Guo Z, Zhang JS. Two-dimensional model for pore pressure accumulations in the vicinity of a buried pipeline. J Offshore Mech. Arct. 2014; 136: 042001.

[11] Seed HB, Rahman MS. Wave-induced pore pressure in relation to ocean floor stability of cohesionless soils. Mar Geotechnol. 1978; 3(2): 123-50.

[12] Rodríguez-Castellanos A, Martínez-Calzada V, Rodríguez-Sánchez JE, Orozco-Del-Castillo M, Carbajal-Romero M. Induced water pressure profiles due to seismic motions. Appl Ocean Res. 2014; 47, 9-16.

[13] Aguirre J, Irikura K. Nonlinearity, liquefaction, and velocity variation of soft soil layers in Port Island, Kobe, during the Hyogo-ken Nanbu earthquake. Bull. Seism. Soc. Am. 1997; 87(5): 1244-1258.

[14] Kagawa T, Sato M, Minowa C, Abe A, Tazoh T. Centrifuge simulations of large-scale shaking table tests: case studies. J. Geotech. Geoenviron. 2004; 130(7): 663-672.

[15] Yang J. Reappraisal of vertical motion effects on soil liquefaction. Géotechnique. 2004; 54(10): 671-676.

[16] Yang J, Sato T, Savidis S, Li XS. Horizontal and vertical components of earthquake ground motions at liquefiable sites. Soil Dyn Earthq Eng. 2002; 22(3): 229-240.

[17] Ye J. Seismic response of poro-elastic seabed and composite breakwater under strong earthquake loading. Bull. Earthquake Engng. 2012; 10(5): 1609-1633.

[18] Ye J, Wang G. Seismic dynamics of offshore breakwater on liquefiable seabed foundation. Soil Dyn Earthq Eng. 2015; 76: 86-99.

[19] Ulker MBC, Rahman MS, Jeng DS. Wave-induced response of seabed: various formulations and their 
applicability. Appl. Ocean. Res. 2009; 31(1): 12-24

[20] Hsu JRC, Jeng DS, Lee CP. Oscillatory soil response and liquefaction in an unsaturated layered seabed. Int. J. Numer. Anal. Methods Geomech. 1995; 19(12): 825-849.

[21] Ulker MBC. Pore pressure, stress distributions, and instantaneous liquefaction of two-layer soil under waves. J. Waterw. Port Coastal Ocean Eng. 2012; 138(6): 435-450.

[22] Wen F, Wang JH. Response of layered seabed under wave and current loading. J. Coastal. Res. 2013; 31(4): 907-919.

[23] Zhou XL, Xu B, Wang JH, Li YL. An analytical solution for wave-induced seabed response in a multi-layered poro-elastic seabed. Ocean Eng. 2011; 38(1): 119-129.

[24] Biot MA. Mechanics of deformation and acoustic propagation in porous media. J. Appl. Phys. 1962; 33(4): 1482-1498.

[25] Zienkiewicz OC, Chang CT, Bettess P. Drained, undrained, consolidating and dynamic behaviour assumptions in soils. Géotechnique. 1980; 30(4): 385-395.

[26] Zienkiewicz OC, Shiomi T. Dynamic behavior of saturated porous media; the generalized Biot formulation and its numerical solution. Int. J. Numer. Anal. Methods Geomech. 1984; 8(1): 71-96.

[27] Okusa S. Wave-induced stresses in unsaturated submarine sediments. Géotechnique. 1985; 35(4): 517- 532.

[28] Rajapakse RKND, Senjuntichai T. Dynamic response of a multi-layered poroelastic medium, Earthquake Eng. Struct. Dyn. 1995; 24: 703-722.

[29] Lee JH, Kim JK. Dynamic response analysis of a floating offshore structure subjected to the hydrodynamic pressures induced from seaquakes. Ocean Eng. 2015; 101: 25-39.

[30] Yang J, Sato T. Interpretation of seismic vertical amplification observed at an array site. Bull. Seismol. Soc. Am. 2000; 90(2): 275-285.

[31] Matsumoto H, Kaneda Y. Some features of bottom pressure records at the 2011 Tohoku earthquake-Interpretation of the far-field DONET data. Proceedings, The $11^{\text {th }}$ Society of Exploration Geophysicists of Japan International Symposium. Japan. 2013; 493-496.

[32] Filloux JH. Tsunami record on the open ocean floor, Geophys. Res. Lett. 1982; 9(1): 25-28.

[33] Levin BW, Nosov MA. Physics of Tsunamis, Springer, Berlin. 2009.

[34] Zhou XL, Jeng DS, Yan YG, Wang JH. Wave-induced multi-layered seabed response around a buried pipeline. Ocean Eng. 2013; 72: 195-208.

[35] Jeng DS. Wave-induced seabed instability in front of a breakwater. Ocean Eng. 1997; 24(10): 887-917. 


\section{APPENDIX :}

2

The following matrix form of coefficients can be derived based the boundary conditions

$$
[M]\left[b_{1}^{(1)}, b_{2}^{(1)}, b_{3}^{(1)}, b_{4}^{(1)}, b_{1}^{(2)}, b_{2}^{(2)}, b_{3}^{(2)}, b_{4}^{(2)}\right]^{T}=[N]
$$

The explicit expressions of the elements $m_{i j}$ of $[M]$ are given as follows

5

$$
m_{11}=\left(\frac{\mathrm{i} k_{p 1}^{(1)}}{n^{(1)} \beta^{(1)}}-\omega^{2} \rho_{f}^{(1)} d\right)\left(1+\eta_{1}^{(1)}\right) e^{-\mathrm{i} k_{p p}^{(1)} L} \quad m_{12}=-\left(\frac{\mathrm{i} k_{p 1}^{(1)}}{n^{(1)} \beta^{(1)}}+\omega^{2} \rho_{f}^{(1)} d\right)\left(1+\eta_{2}^{(1)}\right) e^{i k_{p 1}^{(1)} L}
$$

6

$$
m_{13}=\left(\frac{\mathrm{i} k_{p 2}^{(1)}}{n^{(1)} \beta^{(1)}}-\omega^{2} \rho_{f}^{(1)} d\right)\left(1+\eta_{3}^{(1)}\right) e^{-\mathrm{i} k_{p 2}^{(1)} L} m_{14}=-\left(\frac{\mathrm{i} k_{p 2}^{(1)}}{n^{(1)} \beta^{(1)}}+\omega^{2} \rho_{f}^{(1)} d\right)\left(1+\eta_{4}^{(1)}\right) e^{i k_{p 2}^{(1)} L}
$$

7

$m_{15}=m_{16}=m_{17}=m_{18}=m_{25}=m_{26}=m_{27}=m_{28}=0$

8

$m_{21}=-k_{p 1}^{(1)} e^{-\mathrm{i} k_{p 1}^{(1)} L} \quad m_{22}=k_{p 1}^{(1)} e^{\mathrm{i} k_{p 1}^{(1)} L} \quad m_{23}=-k_{p 2}^{(1)} e^{-\mathrm{ik} k_{p 2}^{(1)} L} \quad m_{24}=k_{p 2}^{(1)} e^{\mathrm{i} e_{p 2}^{(1)} L}$

9

$m_{31}=\frac{\left(1+\eta_{1}^{(1)}\right) k_{p 1}^{(1)}}{n^{(1)} \beta^{(1)}} e^{-i k_{p p}^{(1)} m L} \quad m_{32}=-\frac{\left(1+\eta_{2}^{(1)}\right) k_{p 1}^{(1)}}{n^{(1)} \beta^{(1)}} e^{i k_{p 1}^{(1)} m L}$

10

$m_{33}=\frac{\left(1+\eta_{3}^{(1)}\right) k_{p 2}^{(1)}}{n^{(1)} \beta^{(1)}} e^{-\mathrm{i} k_{p 2}^{(1)} m L} \quad m_{34}=-\frac{\left(1+\eta_{4}^{(1)}\right) k_{p 2}^{(1)}}{n^{(1)} \beta^{(1)}} e^{i k_{p 2}^{(1)} m L}$

11

$m_{35}=-\frac{\left(1+\eta_{1}^{(2)}\right) k_{p l}^{(2)}}{n^{(2)} \beta^{(2)}} e^{-i k_{p 1}^{(2)} m L} \quad m_{36}=\frac{\left(1+\eta_{2}^{(2)}\right) k_{p l}^{(2)}}{n^{(2)} \beta^{(2)}} e^{i k_{p l}^{(2)} m L}$

12

$m_{37}=-\frac{\left(1+\eta_{3}^{(2)}\right) k_{p 2}^{(2)}}{n^{(2)} \beta^{(2)}} e^{-\mathrm{i} k_{p 2}^{(2)} m L} \quad m_{38}=\frac{\left(1+\eta_{4}^{(2)}\right) k_{p 2}^{(2)}}{n^{(2)} \beta^{(2)}} e^{\mathrm{i} k_{p 2}^{(2)} m L}$

13

$m_{43}=-\left(\lambda^{(1)}+2 \mu^{(1)}\right) k_{p 2}^{(1)} e^{-\mathrm{i} k_{p 2}^{(1)} m L} m_{44}=\left(\lambda^{(1)}+2 \mu^{(1)}\right) k_{p 2}^{(1)} e^{\mathrm{i} k_{p 2}^{(1)} m L}$

$m_{45}=\left(\lambda^{(2)}+2 \mu^{(2)}\right) k_{p l}^{(2)} e^{-\mathrm{ik} k_{p l}^{(2)} m L} \quad m_{46}=-\left(\lambda^{(2)}+2 \mu^{(2)}\right) k_{p l}^{(2)} e^{\mathrm{i} k_{p l}^{(2)} m L}$

$m_{47}=\left(\lambda^{(2)}+2 \mu^{(2)}\right) k_{p 2}^{(2)} e^{-\mathrm{ik} k_{p 2}^{(2)} m L} \quad m_{48}=-\left(\lambda^{(2)}+2 \mu^{(2)}\right) k_{p 2}^{(2)} e^{\mathrm{i} k_{p 2}^{(2)} m L}$

$m_{51}=e^{-\mathrm{i} k_{p 1}^{(1)} m L} \quad m_{52}=e^{\mathrm{i} k_{p 1}^{(1)} m L} \quad m_{53}=e^{-\mathrm{i} k_{p 2}^{(1)} m L} m_{54}=e^{\mathrm{i} k_{p 2}^{(1)} m L}$

$m_{55}=-e^{-\mathrm{i} k_{p l}^{(2)} m L} \quad m_{56}=-e^{\mathrm{i} k_{p l}^{(2)} m L} \quad m_{57}=-e^{-\mathrm{i} k_{p 2}^{(2)} m L} \quad m_{58}=-e^{\mathrm{i} k_{p 2}^{(2)} m L}$

$m_{61}=\eta_{1}^{(1)} e^{-\mathrm{i} k_{p 1}^{(1)} m L} \quad m_{62}=\eta_{2}^{(1)} e^{\mathrm{i} \kappa_{p 1}^{(1)} m L} \quad m_{63}=\eta_{3}^{(1)} e^{-\mathrm{i} k_{p 2}^{(1)} m L} \quad m_{64}=\eta_{4}^{(1)} e^{\mathrm{i} k_{p 2}^{(1)} m L}$ 


$$
m_{65}=-\eta_{1}^{(2)} e^{-\mathrm{i} k_{p 1}^{(2)} m L} \quad m_{66}=-\eta_{2}^{(2)} e^{\mathrm{i} k_{p 1}^{(2)} m L} \quad m_{67}=-\eta_{3}^{(2)} e^{-\mathrm{i} k_{p 2}^{(2)} m L} \quad m_{68}=-\eta_{4}^{(2)} e^{\mathrm{i} k_{p 2}^{(2)} m L}
$$

$2 \quad m_{75}=m_{76}=m_{77}=m_{78}=1$

$3 \quad m_{71}=m_{72}=m_{73}=m_{74}=m_{81}=m_{82}=m_{83}=m_{84}=0$

$4 \quad m_{85}=\eta_{1}^{(2)} \quad m_{85}=\eta_{1}^{(2)} \quad m_{87}=\eta_{3}^{(2)} \quad m_{88}=\eta_{4}^{(2)}$

5 The elements in $[N]$ are given by

6

$$
n_{1}=n_{2}=n_{3}=n_{4}=n_{5}=n_{6}=n_{8}=0 \quad n_{7}=u_{0}
$$




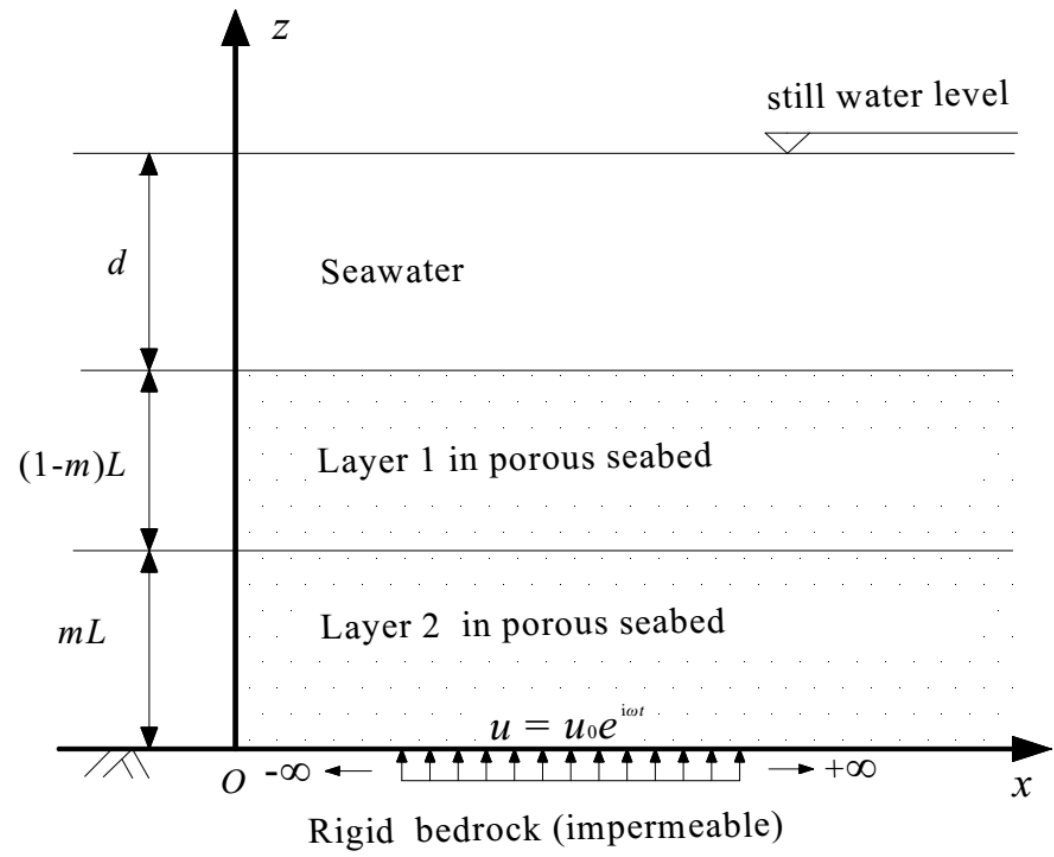

Fig. 1. Two-layer porous seabed under vertical seismic action. 


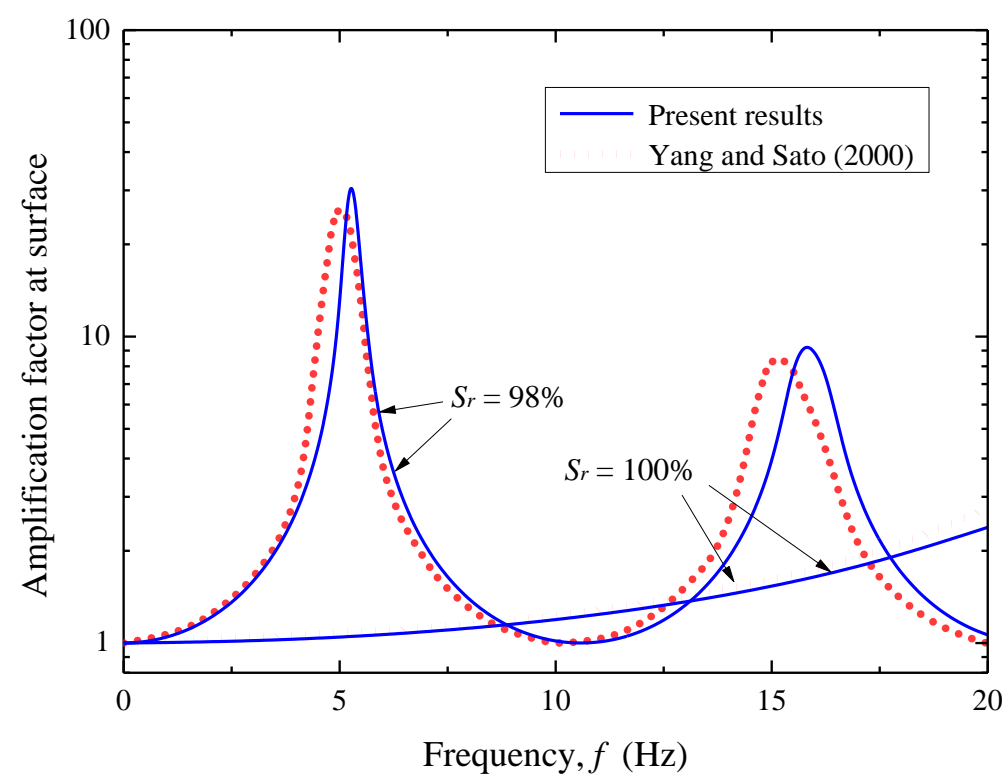

4

5 Fig. 2. Effects of frequency and saturation degree on the amplification factor of displacement at 6 seabed surface when the seawater is removed (i.e., $d=0 \mathrm{~m}$ ) 
(a)

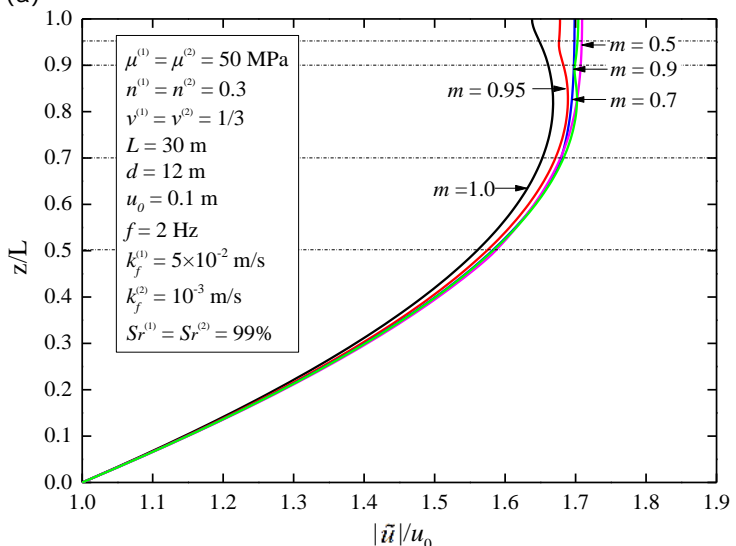

(c)

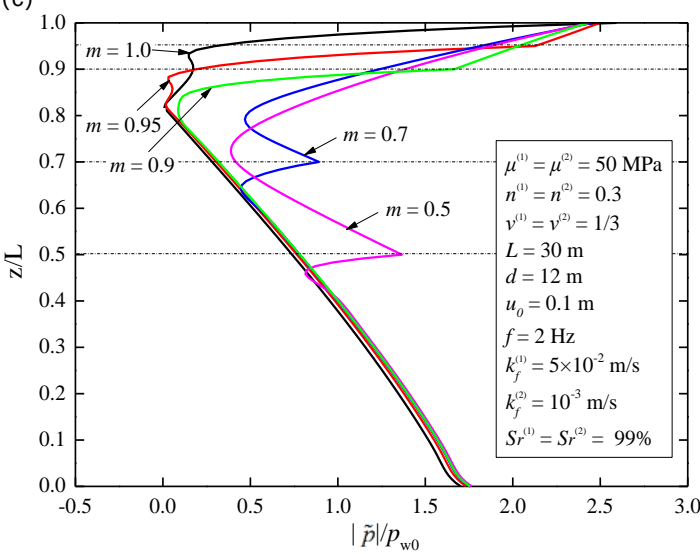

(b)

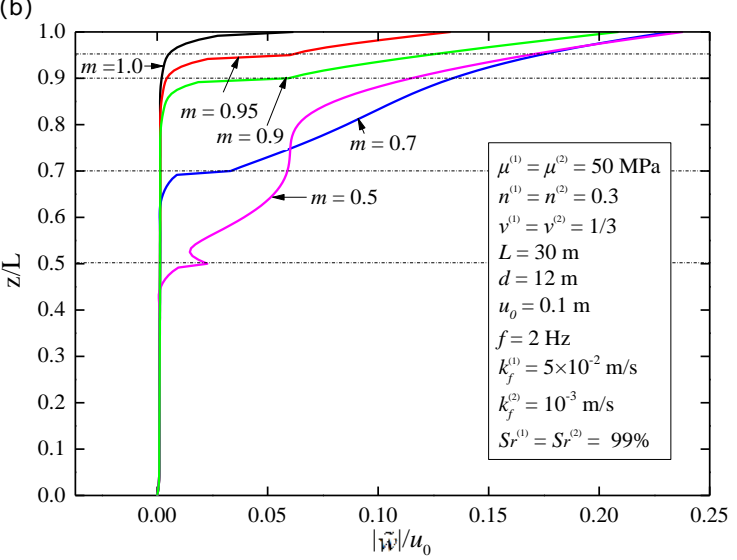

(d)

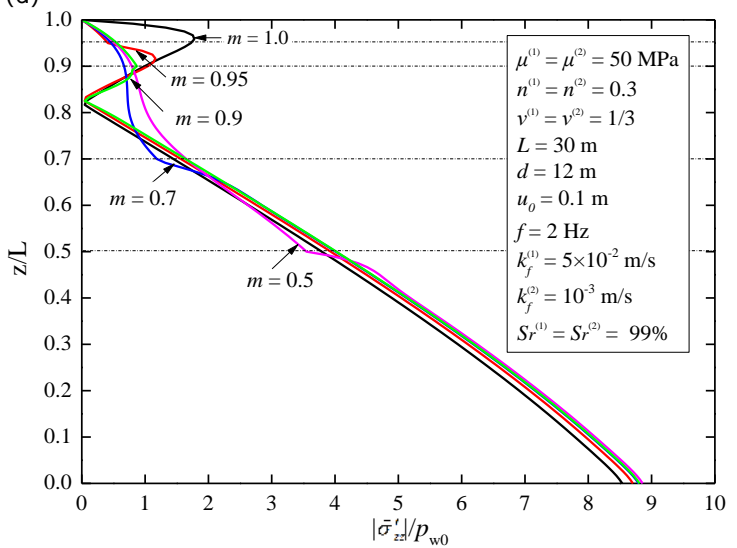

Fig. 3. Distribution of (a) soil displacement $|\tilde{u}| / u_{0}$ (b) relative fluid displacement $|\tilde{w}| / u_{0}$ (c) pore pressure $|\tilde{p}| / p_{w 0}$ (d) vertical effective stress $\left|\tilde{\sigma}_{z z}^{\prime}\right| / p_{w 0}$ versus relative depth $(z / L)$ for various relative thickness ratios. 
(a)

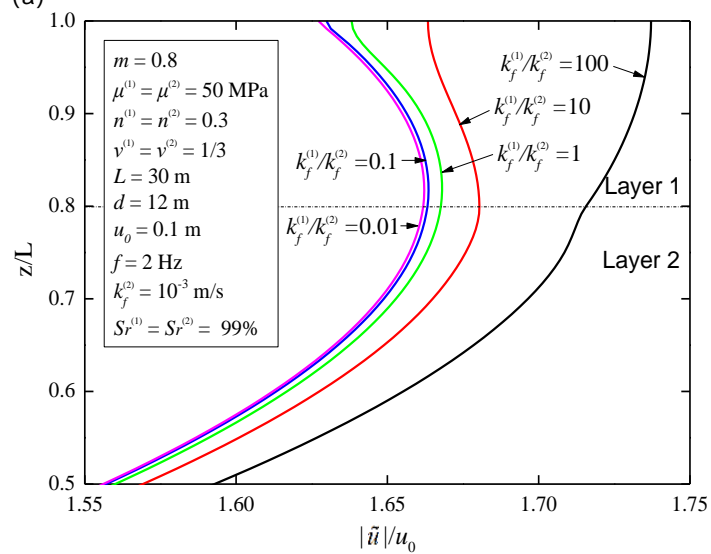

(c)

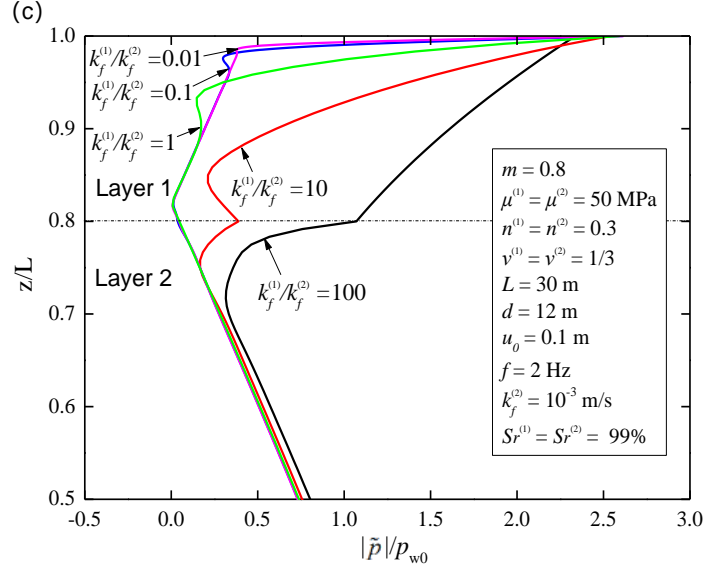

(b)

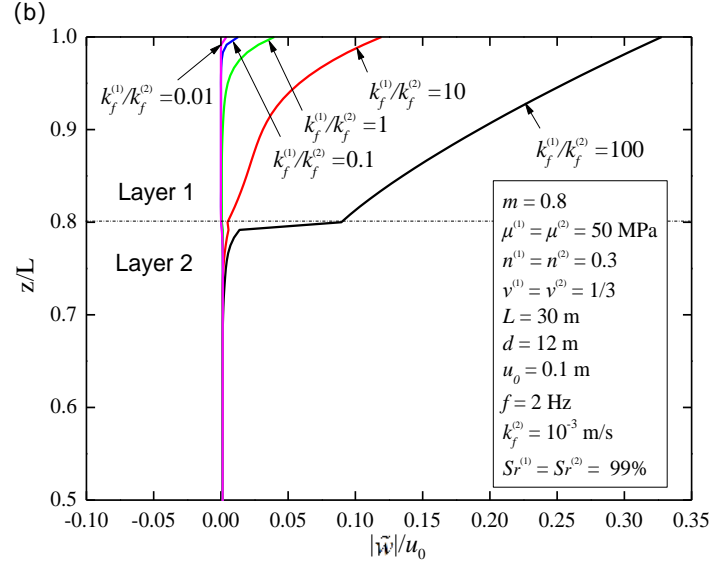

(d)

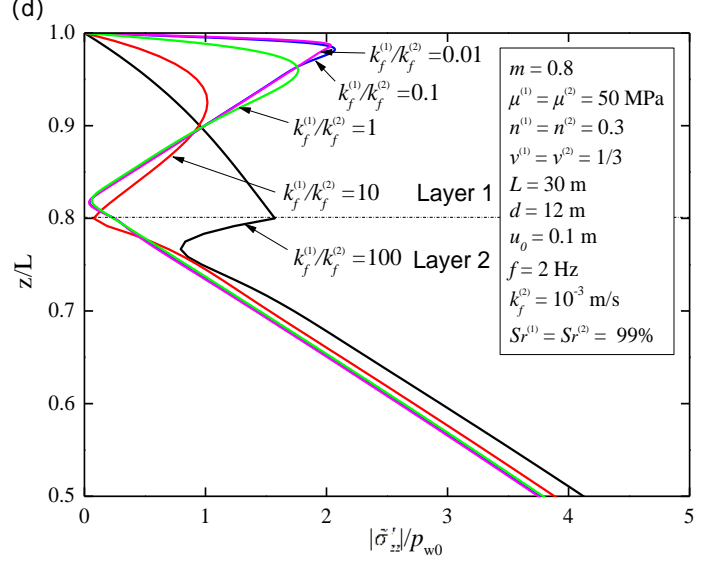

Fig. 4. Distribution of (a) soil displacement $|\tilde{u}| / u_{0}$ (b) relative fluid displacement $|\tilde{w}| / u_{0}$ (c) pore pressure $|\tilde{p}| / p_{w 0}$ (d) vertical effective stress $\left|\tilde{\sigma}_{z z}^{\prime}\right| / p_{w 0}$ versus relative depth $(z / L)$ for various relative permeabilities. 
(a)

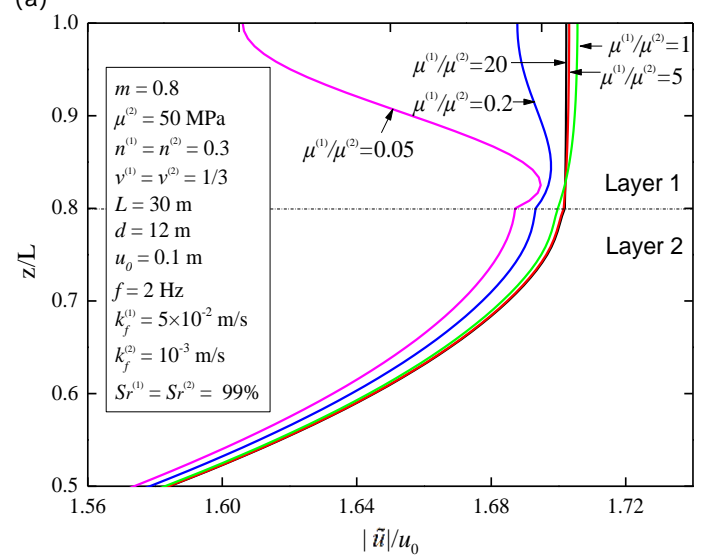

(c)

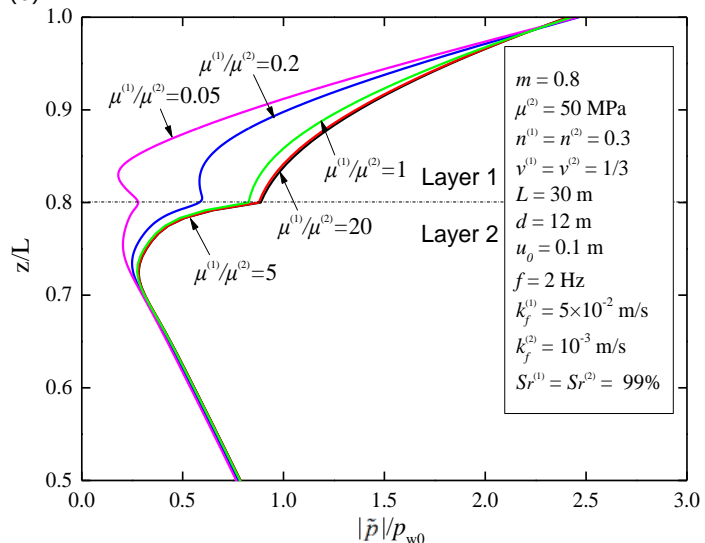

(b)

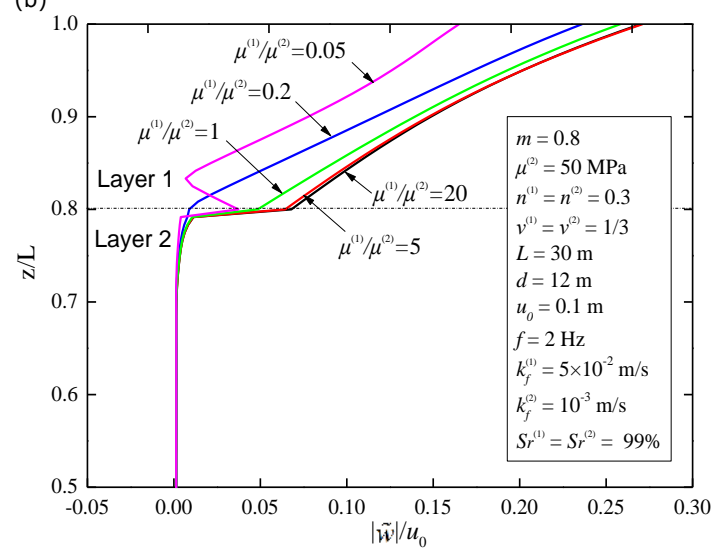

(d)

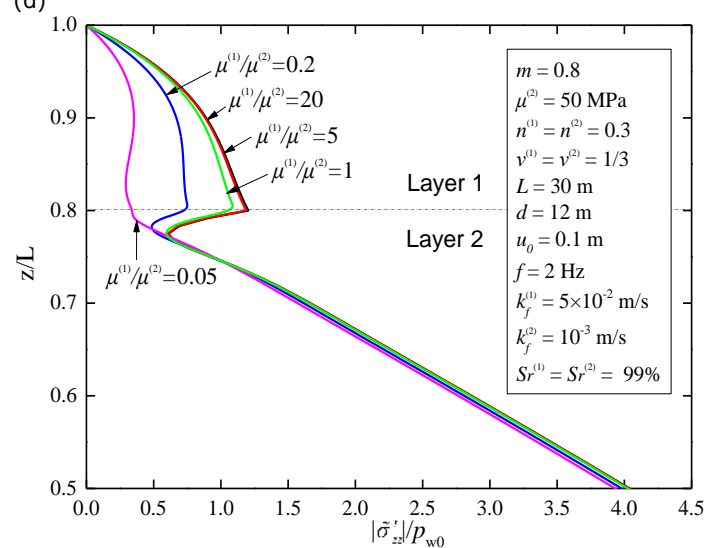

Fig. 5. Distribution of (a) soil displacement $|\tilde{u}| / u_{0}$ (b) relative fluid displacement $|\tilde{w}| / u_{0}$ (c) pore pressure $|\tilde{p}| / p_{w 0}$ (d) vertical effective stress $\left|\tilde{\sigma}_{z z}^{\prime}\right| / p_{w 0}$ versus relative depth $(z / L)$ for various relative shear moduli. 
(a)

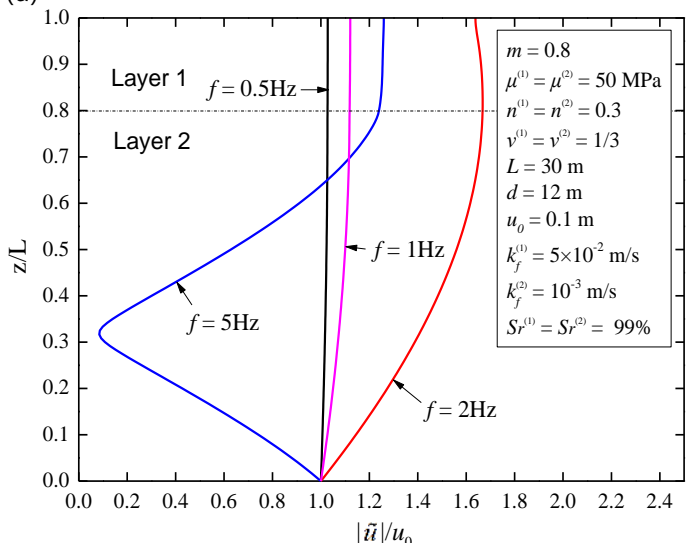

(c)

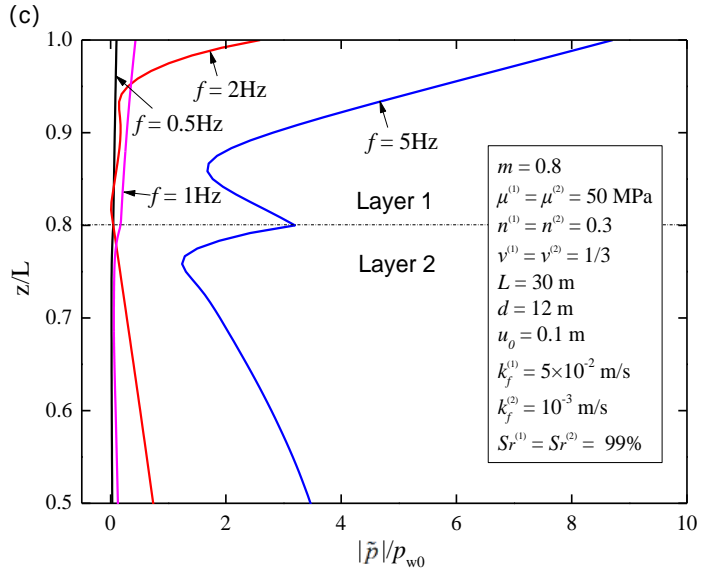

(b)

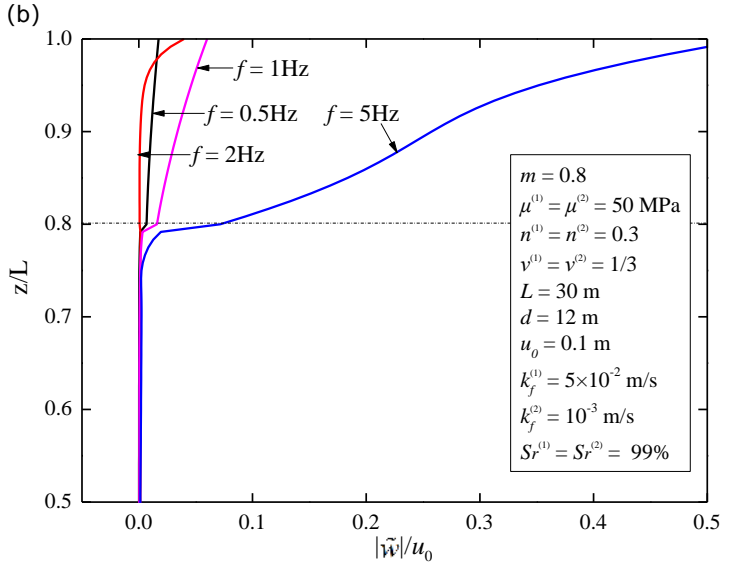

(d)

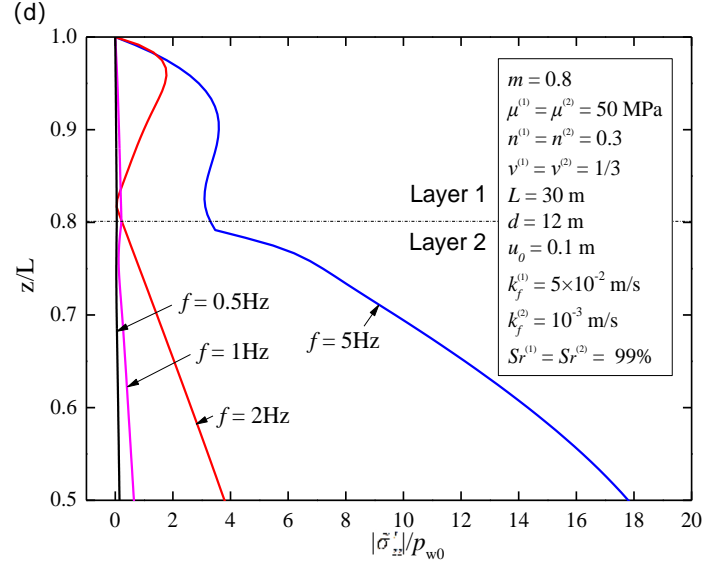

Fig. 6. Distribution of (a) soil displacement $|\tilde{u}| / u_{0}$ (b) relative fluid displacement $|\tilde{w}| / u_{0}$ (c) pore pressure $|\tilde{p}| / p_{w 0}$ (d) vertical effective stress $\left|\tilde{\sigma}_{z z}^{\prime}\right| / p_{w 0}$ versus relative depth $(z / L)$ for various earthquake frequencies. 

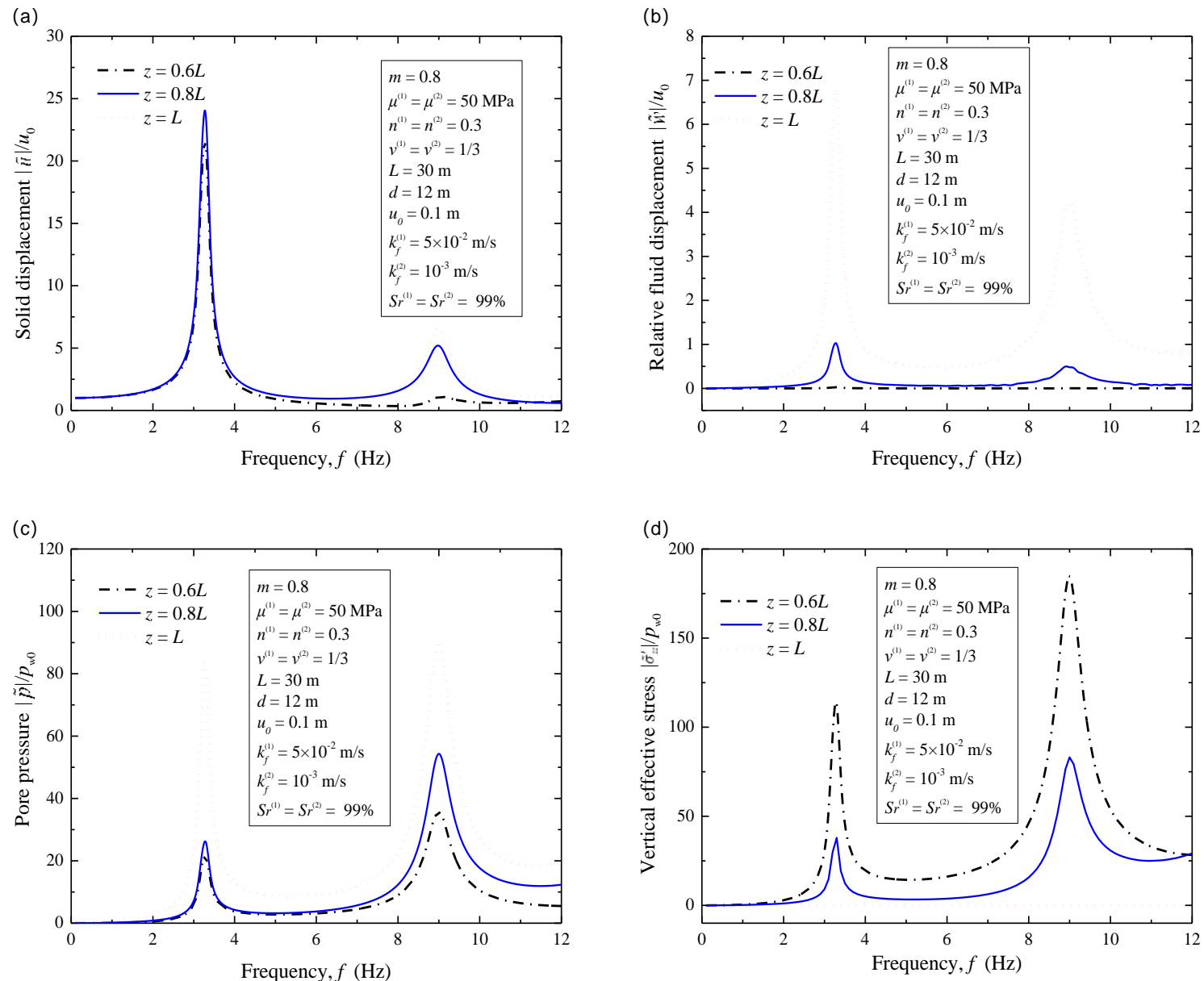

Fig. 7. Transfer functions of induced responses in various relative depths of seabed: (a) soil displacement,

(b) relative fluid displacement, (c) pore pressure, and (d) vertical effective stress. 


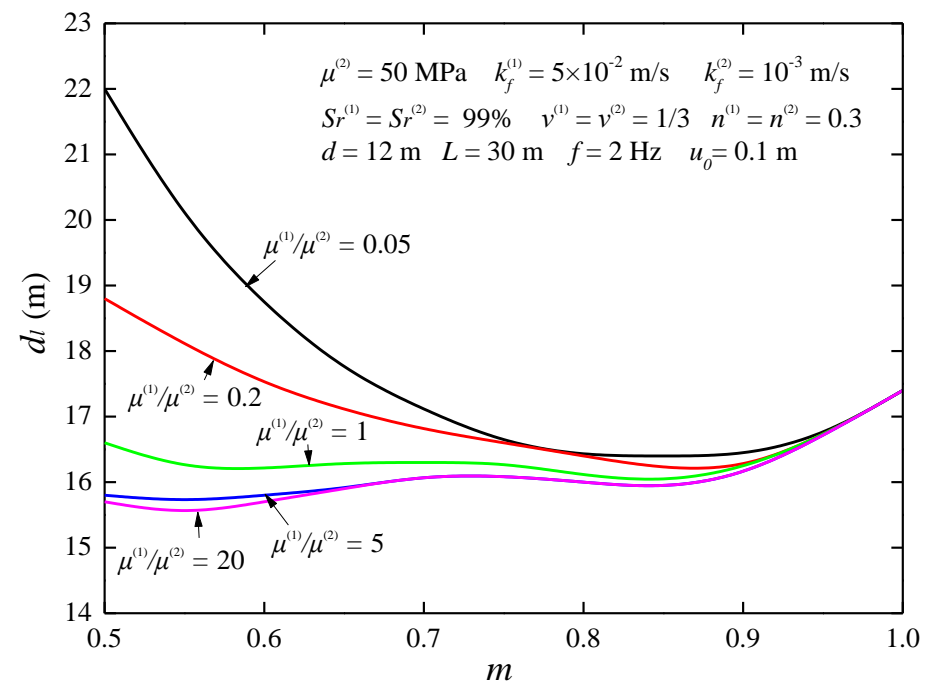

Fig. 8. Relationship between maximum liquefaction depth $d_{l}$ and relative thickness ratio $m$ for various relative shear moduli.

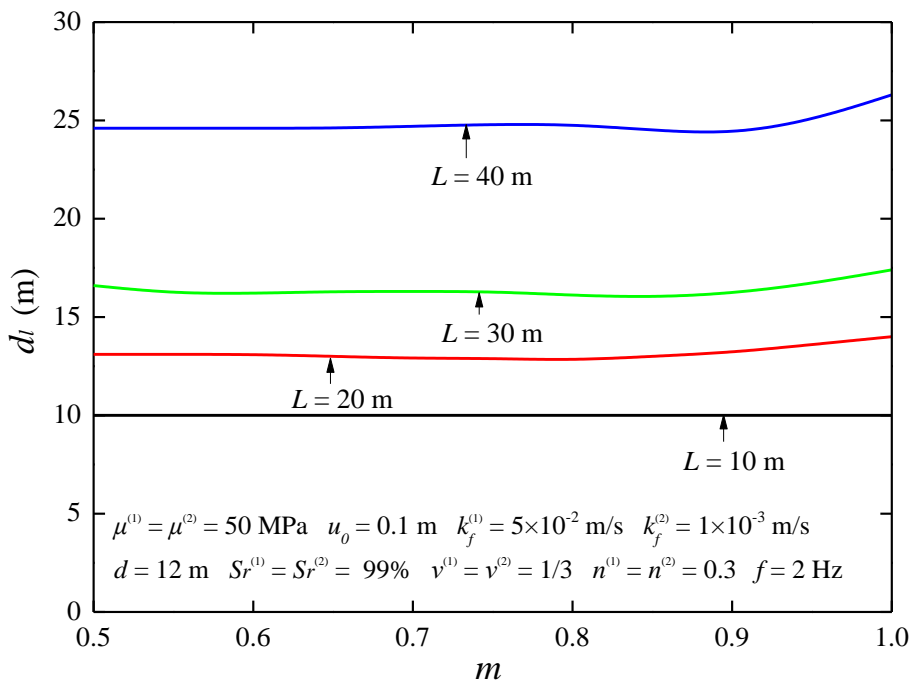

Fig. 9. Relationship between maximum liquefaction depth $d_{l}$ and relative thickness ratio $m$ for various thicknesses of seabed. 


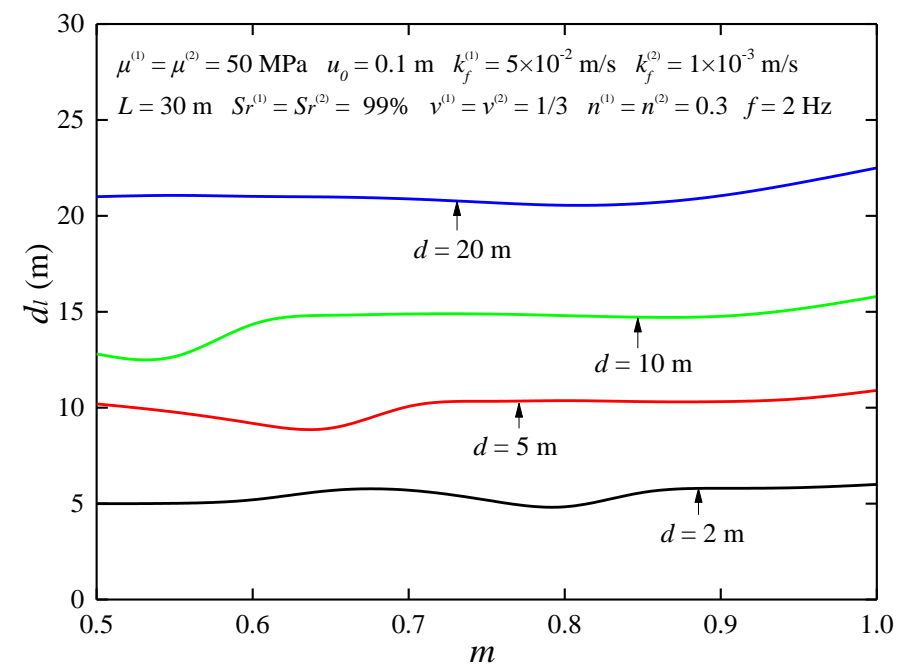

Fig. 10. Relationship between maximum liquefaction depth $d_{l}$ and relative thickness ratio $m$ for various depths of seawater.

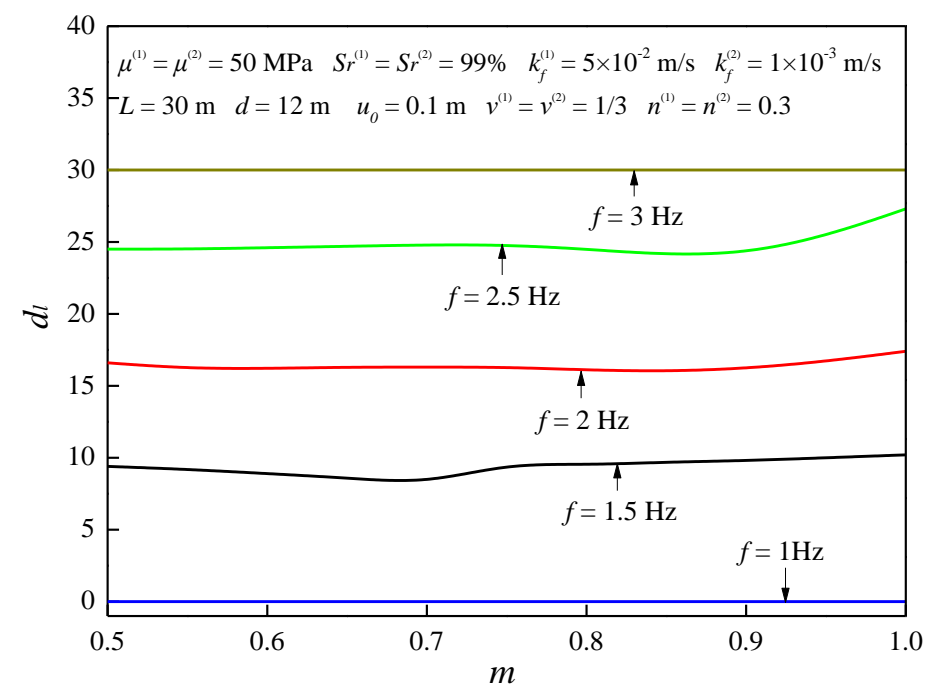

Fig. 11. Relationship between maximum liquefaction depth $d_{l}$ and relative thickness ratio $m$ for various earthquake frequencies. 

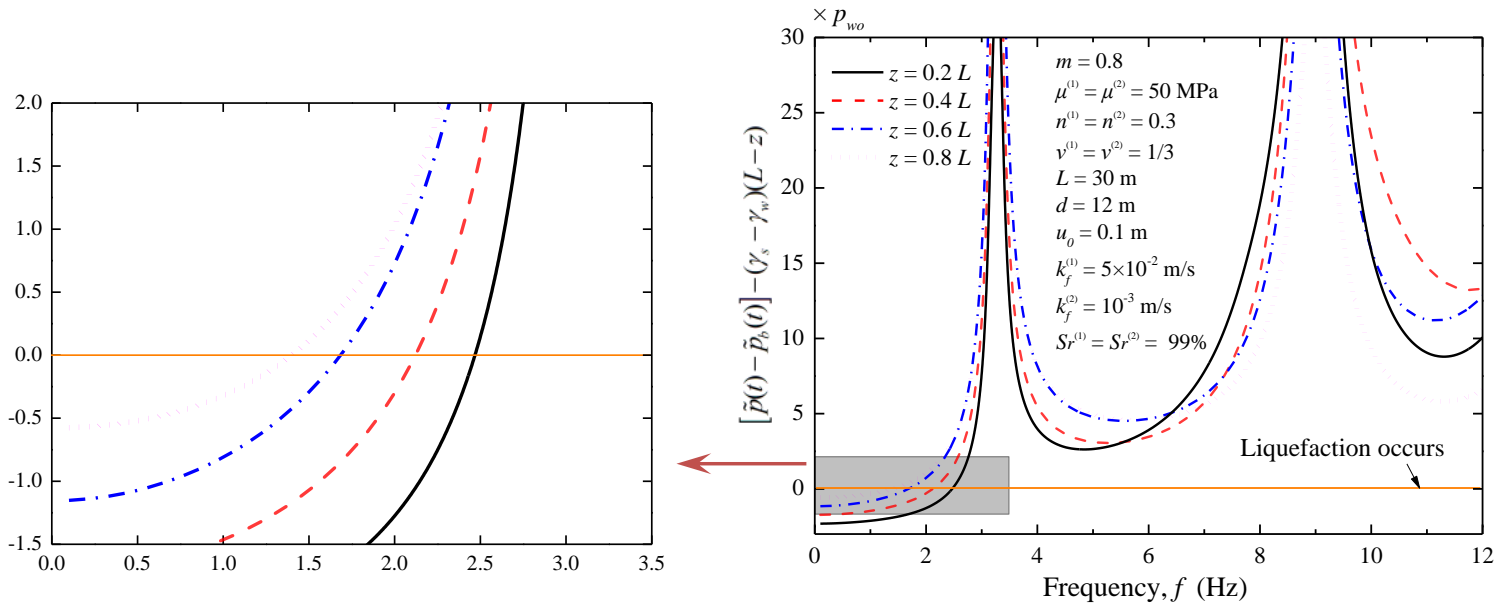

Fig. 12. Variation of $\left[\tilde{p}(t)-\tilde{p}_{b}(t)\right]-\left(\gamma_{s}-\gamma_{w}\right)(L-z)$ with frequency $f$ at different depths of the seabed. 


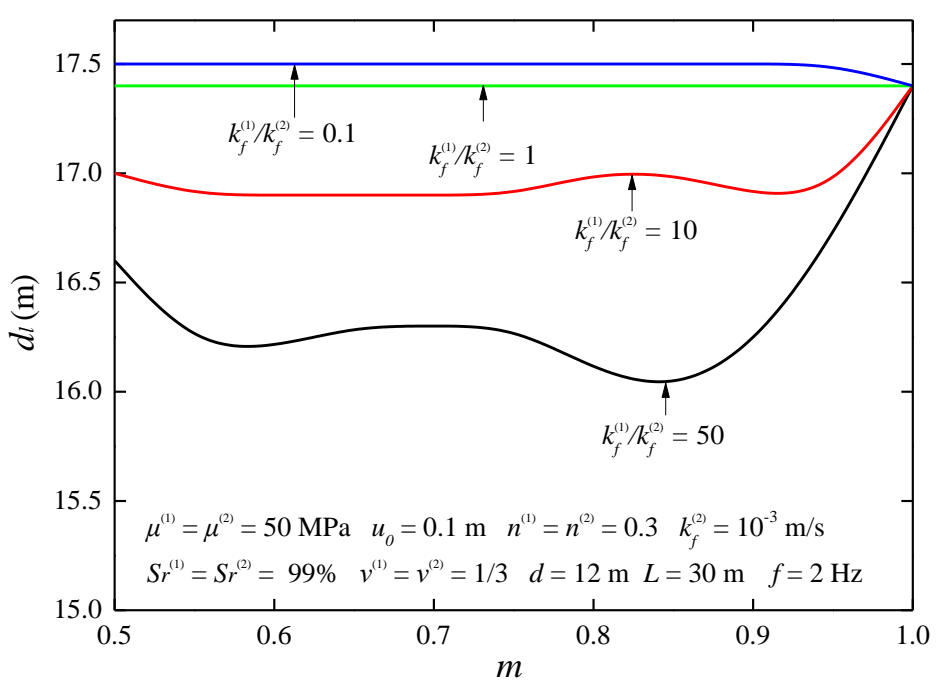

Fig. 13. Relationship between maximum liquefaction depth $d_{l}$ and relative thickness ratio $m$ for various relative permeabilities.

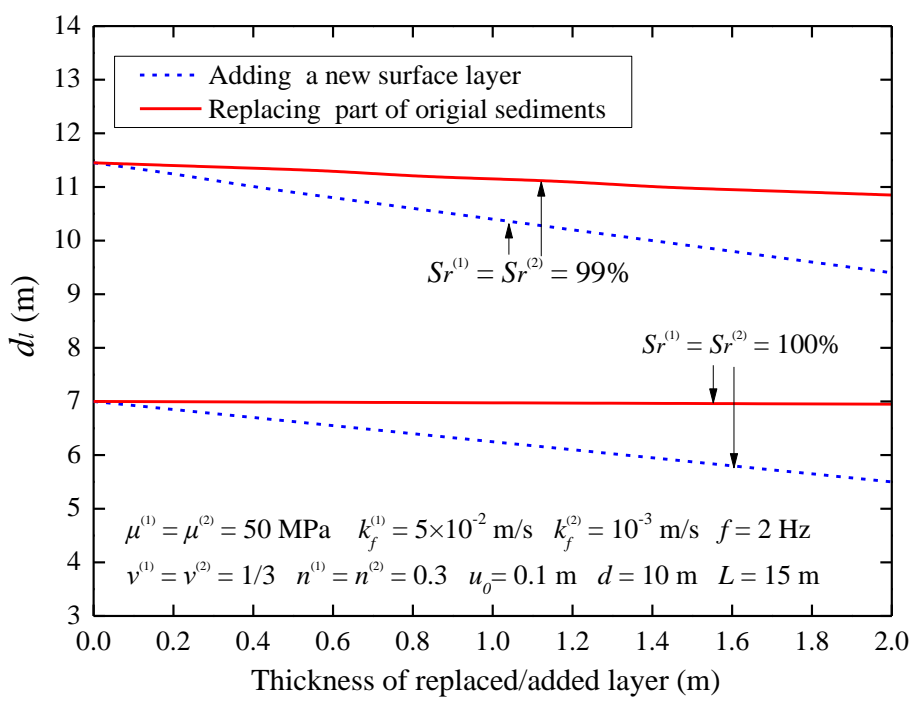

Fig. 14. Relationship between maximum liquefaction depth $d_{l}$ and layer thickness using two different treatments. 